\section{Addressing the psychosocial needs of genital herpes sufferers}

Improved patient management must be a priority if we are to reduce the adverse consequences that genital herpes may have on affected individuals and also the contribution this common cause of genital ulceration makes to the transmission of HIV infection.

Many patients experience frequently recurring episodes from the life-long infection which follows first episodes. It has long been recognised that psychological factors, such as emotional stress, can trigger recurrences. In addition, it is clear that the diagnosis of herpes brings with it a psychological burden which impinges on normal social and sexual functioning. ${ }^{12}$ Patients feel controlled by the virus and are often afraid to discuss their predicament with partners and friends for fear of derision and rejection. ${ }^{3}$

To provide effective management of genital herpes, physicians must address both the medical and psychosocial needs of their patients. At the first diagnosis of genital herpes, patients should be educated about the nature, features and transmission of their infection and individually assessed to determine its likely psychosocial impact. Similarly, patients with frequently recurring or severe disease often need additional counselling and support as part of their overall disease management.

Effective reassurance and counselling, together with optimal medical management of symptoms, can help to reduce the frequency of recurrences and allow patients relief from the psychological distress of genital herpes. ${ }^{45}$ Clearly, in patients whose quality of life is significantly impaired by frequent recurrences, suppressive treatment with acyclovir can provide effective control of symptoms. ${ }^{6}$ However, the use of antiviral treatment should be as an adjunct to patient education, counselling and support rather than as a replacement for it.

Patient denial, lack of knowledge and inadequate counselling contribute to spread of disease. It is essential that consultations provide sufficient time to counteract these problems. Educational sessions can also help patients with subclinical infection to identify manifestations of recurrences, when the risk of transmission to sexual partners is greatest. ${ }^{7}$

Both primary care and specialist physicians are involved in managing patients with genital herpes, and depending on the particular health care system in operation, the job of counselling and providing psychological support may fall to the physician or to another trained healthcare professional. Whatever the division of labour between health-care professionals, it is essential that those at the first point of contact with patients do not overlook the psychological needs integral to the disease and its management. In genitourinary medicine clinics, this requirement should be considered as a measure of service quality which has important planning and resource implications.
1 Woolley PD, Kinghorn GR, The psychological aspects of genital herpes. Stress Medicine 1986;2:193-5.

Goldmeier D, Johnson A, Byrne M, Barton S. Psychologica implications of recurrent genital herpes simplex virus infection. Genitourin Med 1988;64:527-30.

3 Rose FB, Camp CJ. Genital herpes: how to relieve patients physical and psychological symptoms. Postgraduate Megi cine $1988 ; 84: 81-6$

4 Vanderplate C, Aral SO, Magder L. The relationship ame्सेg genital herpes simplex virus, stress, and social support. Health Psychology 1988;7:159-68.

5 Drob S, Loemer M, Lifshutz H. Genital herpes: psychological consequences. Br f Med Psychol 198 58:307-15.

6 Carney O, Mindel A, Ikkos G, Williams P. The effects of suppressive oral acyclovir on psychological morbidity patients with frequently recurring genital herpes. MSSVD May 1990

7 Langenberg A, Benedetti J, Jenkins J, et al. Developmen ${ }_{0}$ clinically recognisable genital lesions among wonf previously identified as having asymptomatic herfes simplex virus type 2 infection. Ann Intern Med 19\% simplex virus $110: 882-7$.

Accepted for publication 14 July 1992.

Aphthous ulcers in HIV-infected patient: treatment with thalidomide

Aphthous ulcerations in HIV-infected perso每s can be severe and persistent. ${ }^{1}$ They may involve the genital area, gastrointestinal tract, as well as the mouth and pharynx. Ulcerations caused by fungal, bacterial or viral agents and malignancy must be excluded before idiopathic aphthous ulcerations are considered.

In recurrent aphthous ulcers, immunosu pressive and cytotoxic agents are often used, but these agents may be dangerous in alrea debilitated patients. We report a case of HIV-infected patient with aphthous ulce⿶s which dramatically improved within 48 hous of thalidomide treatment.

A 25 year old homosexual man, HIDI] antibody positive since 1988 , was admitted 1992 for fever, discrete generalized erythema, dysphagia, pharyngitis and myalgia. Clinigigl examination showed oral aphthous ulces, bilateral conjunctivitis and painful aphthogd ulcerated lesions of the scrotum $(2-3 \mathrm{~cm}$ diameter) (fig). Mild inguinal, axillary amd cervical lymphadenopathy was present. evidence of viral, bacterial or fungal infection

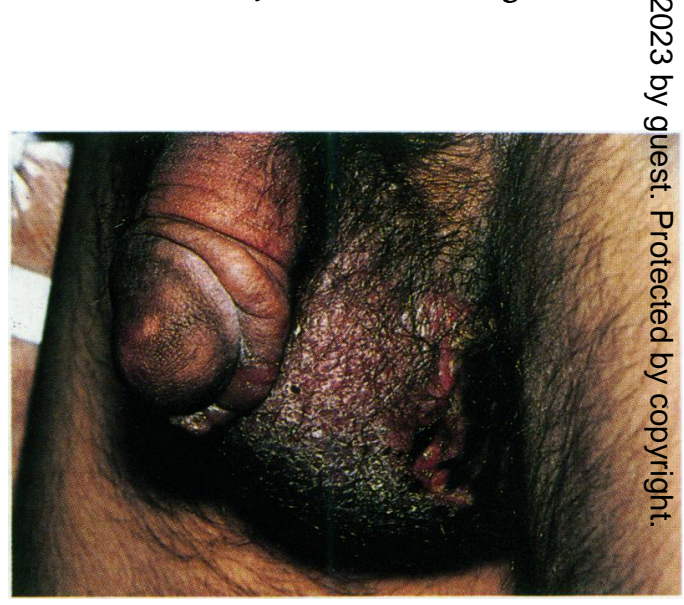

Figure Large painful aphthous ulcer of the scrotum. 